\title{
The influence of broken cloudiness on cloud top height retrievals using nadir observations of backscattered solar radiation in the oxygen A-band
}

\author{
A.A. Kokhanovsky,*, B. Mayer ${ }^{\mathrm{b}}$, V.V. Rozanov ${ }^{\mathrm{a}}$, K. Wapler ${ }^{\mathrm{b}}$, \\ J.P. Burrows ${ }^{\mathrm{a}}$, U. Schumann ${ }^{\mathrm{b}}$ \\ ${ }^{a}$ Institute of Environmental Physics, University of Bremen, Otto Hahn Allee 1, D-28334, Bremen, Germany \\ ${ }^{\mathrm{b}}$ Institute of Atmospheric Physics, Deutsches Zentrum für Luft- und Raumfahrt (DLR), Oberpfaffenhofen, D-82234 Wessling, Germany
}

Received 17 May 2006; received in revised form 13 June 2006; accepted 16 June 2006

\begin{abstract}
The paper is devoted to theoretical studies of the influence of cloud inhomogeneities on cloud top height (CTH) retrievals based on top-of-atmosphere nadir reflectance observations in the oxygen A-band. A three-demensional (3D) Monte Carlo code is used to simulate highly resolved spectral measurements in the oxygen A-band. These synthetic radiances are used as input for the retrieval code SACURA based on asymptotic radiative transfer theory and the independent pixel approximation. The results show that the effect of cloud inhomogeneity on the derived CTHs is small. While we found considerable 3D effects in the reflectance of more than $30 \%$ compared to the independent column approximation, the spectral dependence of the difference was small. As SACURA is mainly based on spectral ratios, the retrieval results are hardly affected by the large absolute deviations. In consequence, SACURA is capable to retrieve CTHs with an accuracy of better than $1.5 \mathrm{~km}$ for overcast and also most partially cloudy cases.
\end{abstract}

(C) 2006 Elsevier Ltd. All rights reserved.

Keywords: Cloud; Remote sensing; Cloud top height; Light scattering; Radiative transfer

\section{Introduction}

The aim of this paper is to study the influence of cloud horizontal inhomogeneity (e.g., broken cloud fields) on the retrieval of cloud parameters using Global Ozone Monitoring Experiment (GOME) and Scanning Imaging Absorption Spectrometer for Atmospheric CHartographY (SCIAMACHY) (e.g., [1,3]). These instruments were conceived to yield global distributions of atmospheric constituents and thereby improve the knowledge of a variety of regional and global issues of importance for the chemistry and physics of the Earth's atmosphere. In addition to trace gas columns, they allow the quantitative determination of the key components of the hydrological cycle, water vapor and clouds, with one single instrument.

\footnotetext{
${ }^{*}$ Corresponding author. Tel./fax: +49421218 .

E-mail address: alexk@iup.physik.uni-bremen.de (A.A. Kokhanovsky).
} 
To achieve the required combination of high spectral resolution and high radiometric accuracy, a large instantaneous field of view (IFOV) is required, resulting in a pixel size of $40 \times 320 \mathrm{~km}^{2}$ for GOME and $30 \times 60 \mathrm{~km}^{2}$ (at minimum) for SCIAMACHY. As a result of the large IFOV, a correct treatment of cloud inhomogeneity is vital to exploit the full potential of GOME and SCIAMACHY. However, current remote sensing methods for trace gases and aerosols generally assume either cloudless sky or a homogeneous, planeparallel cloud cover. In particular, cloud property retrievals explicitly or implicitly assume horizontal homogeneity of the cloud field within the IFOV of the instrument. On the other hand it is well known that clouds are highly inhomogeneous on all spatial scales, ranging from few centimeters up to the synoptic scale of several thousand kilometers.

This paper is mainly devoted to the study of the influence of sub-pixel cloud inhomogeneity on cloud top height $(\mathrm{CTH})$ retrievals based on top-of-atmosphere (TOA) nadir reflectance measurements in the oxygen A-band (wavelength $\lambda=755-775 \mathrm{~nm}$ ). The case of multi-layered cloudiness is excluded from our analysis. A review of modern techniques to retrieve CTHs is given by Rozanov and Kokhanovsky [32]. The independent pixel approximation (IPA) of the radiative transfer theory is commonly used for the determination of CTH. The IPA assumes that the reflectance of a broken cloud field can be calculated as a weighted average of 1D calculations for the cloudy and cloud-free parts. In particular, the IPA has been applied for the determination of CTH by $[9,11-13,18,20]$, to name only a few. However, these authors just applied IPA for the problem at hand but they did not study its accuracy. Clearly, the applicability of the IPA for the determination of CTH and errors due to the usage of this approximation can be studied only with three-dimensional (3D) radiative transfer calculations of TOA reflectance. For our application we need spectrally resolved 3D calculations of the reflectance in the oxygen A-band. To our knowledge, such calculations have not been performed for broken cloud conditions so far. We used a well-characterized and tested 3D radiative transfer model (RTM) to calculate TOA reflectance in the oxygen A-band. This way we simulated satellite observations for a set of idealized but typical cloud scenarios and checked the applicability of the IPA for the determination of cloud altitudes from space.

In the next two sections we describe the forward and inverse models used in this study. The fourth section discusses the uncertainty of CTH retrievals for partially cloudy scenes.

\section{The forward model}

\subsection{MYSTIC}

The forward radiative transfer calculations in this paper have been performed using the 3D Monte Carlo code for the phYSically correct Tracing of photons In Cloudy atmospheres, MYSTIC [22,23]. MYSTIC is a forward Monte Carlo code which traces photons on their individual paths through a vertically and horizontally inhomogeneous atmosphere. The code is based on an approach similar to that described by Cahalan et al. [4]. Radiances are calculated using the local estimate technique (e.g., [6,21]). MYSTIC has been successfully validated in the intercomparison of 3D radiation codes I3RC [5]. It is operated as part of the libRadtran package [25] which prepares the input properties for the solver and processes the output. libRadtran provides several radiative transfer equation solvers and allows comparisons between those using identical input conditions. This way we found an agreement with the well-tested DISORT code [35] of better than $0.1 \%$ for various $1 \mathrm{D}$ cases. Excellent agreement for a 3D case very similar to this paper was also found by comparison with the RADUGA code, a successive orders of scattering approach for the radiative transfer equation, see plots in Nikolaeva et al. [29]. As for any statistical model, the Monte Carlo results are affected by statistical noise which decreases with the square root of the number of photons. The accuracy which can be reached therefore depends on the available computing power.

For the application in this paper, we assumed a single layer cloud embedded in an atmosphere with vertically varying aerosol scattering and absorption characteristics (see next section). To confirm the performance of MYSTIC for the cases studied in this paper, we compared the results of this code with other radiative codes such as SCIATRAN [31] and RADUGA [29]. 


\subsection{Radiance simulations in the oxygen A-band}

To consider absorption in the oxygen A-band, a correlated-k approximation is required. For that purpose we use the approach of SCIATRAN which has been designed as the forward model for the retrieval of atmospheric constituents from SCIAMACHY [1]. Version 2.0 is used in this paper, see Rozanov et al. [31].

The SCIATRAN RTM is the improved version of the well-known GOMETRAN model which was originally developed to simulate solar radiation backscattered from the atmosphere and reflected from the Earth's surface in the spectral range $240-800 \mathrm{~nm}$ as measured by GOME in nadir viewing geometry. SCIATRAN was extended to $240-2380 \mathrm{~nm}$ covering the full SCIAMACHY spectral range. A new generation of the SCIATRAN model (version 2.0) comprises all features of SCIATRAN 1.2 and supports additionally radiative transfer calculations in a spherical atmosphere. The wavelength range has been extended to $175 \mathrm{~nm}$ including the Schumann-Runge and Herzberg absorption bands of oxygen. The code is freely available for non-commercial purposes at www.iup.physik.uni-bremen.de/sciatran. The SCIATRAN 2.0 radiative transfer solver is based on the discrete ordinate approach similar to that described by Siewert [34].

For our application all relevant atmospheric processes were considered: Rayleigh scattering, aerosol scattering and absorption, molecular absorption, and cloud scattering. A surface albedo of 0 was assumed for the calculations which is a good choice over dark surfaces such as water. The model atmosphere contained 4 aerosol layers, from 0-2, 2-10, 10-30, and 30-60 km (boundary layer, tropospheric, lower and upper stratospheric) with different aerosol properties attributed to each layer as specified by Kneizys et al. [10]. The vertically integrated aerosol optical thickness $\tau$ was set to 0.26 at $760 \mathrm{~nm}$. A Henyey-Greenstein phase function $p(\theta)=\left(1-g^{2}\right)\left(1-2 g y+g^{2}\right)^{-3 / 2}$ was used for the aerosol. Here $y$ is the cosine of the scattering angle $\theta$ and $g$ is the asymmetry parameter assumed to be 0.6 in this work. Due to the weaker scattering of light by aerosols compared to clouds, assumptions on the aerosol characteristics (in particular optical thickness, single scattering albedo, and asymmetry parameter) do not affect the conclusions reached in this paper.

Water clouds with different geometrical and optical thicknesses were positioned at various levels in the atmosphere. A constant cloud scattering phase function was assumed which was calculated using Mie theory at $760 \mathrm{~nm}$ assuming the following droplet size distribution [15]: $f(a)=A a^{6} \exp (-1.5 a)$, where $A=(3 / 2)^{7} / 6$ ! is a normalization constant and $a$ is the droplet radius. The refractive index of water was set to 1.33 in the Mie calculations. Molecular absorption was considered using a five-point correlated $k$-distribution [2] with a spectral resolution of $0.05 \mathrm{~nm}$, based on the HITRAN database [30]. Further details of the model used are given by Kokhanovsky and Rozanov [16].

To assure accurate results in the oxygen A-band, we compared results of MYSTIC and SCIATRAN at the full spectral resolution of $0.05 \mathrm{~nm}$ in Fig. 1 for a homogeneous cloud with optical thickness $\tau=20$. Except for the statistical noise of the Monte Carlo model, good agreement is found between both codes. The systematic difference is very small and negligible for our application. We conclude that for $1 \mathrm{D}$ cases both models produce consistent results which is a pre-requisite for this study.

\section{The inverse model}

SACURA [15,32,33] has been developed to retrieve cloud top altitudes from TOA nadir reflectance observations. Results of retrievals for all SCIAMACHY data acquired globally to this date are freely available at www.iup.physik.uni-bremen.de. SACURA is based on the representation of the TOA reflectance $R$ by a Taylor expansion with respect to the CTH $h$ :

$$
R(h)=R\left(h_{0}\right)+\sum_{i=1}^{\infty} a_{i}\left(h-h_{0}\right)^{i},
$$

where $a_{i}=R^{(i)}\left(h_{0}\right) / i$ ! and $R^{(i)}\left(h_{0}\right)$ is the $i$-derivative of $R$ at height $h_{0}$. To allow inversion, the equation needs to be linearized [32]. We found that the function $R(h)$ is linear over a wide range of $h$ [16] for which reason we neglect the nonlinear terms in Eq. (1):

$$
R(h)=R\left(h_{0}\right)+R^{\prime}\left(h_{0}\right)\left(h-h_{0}\right),
$$



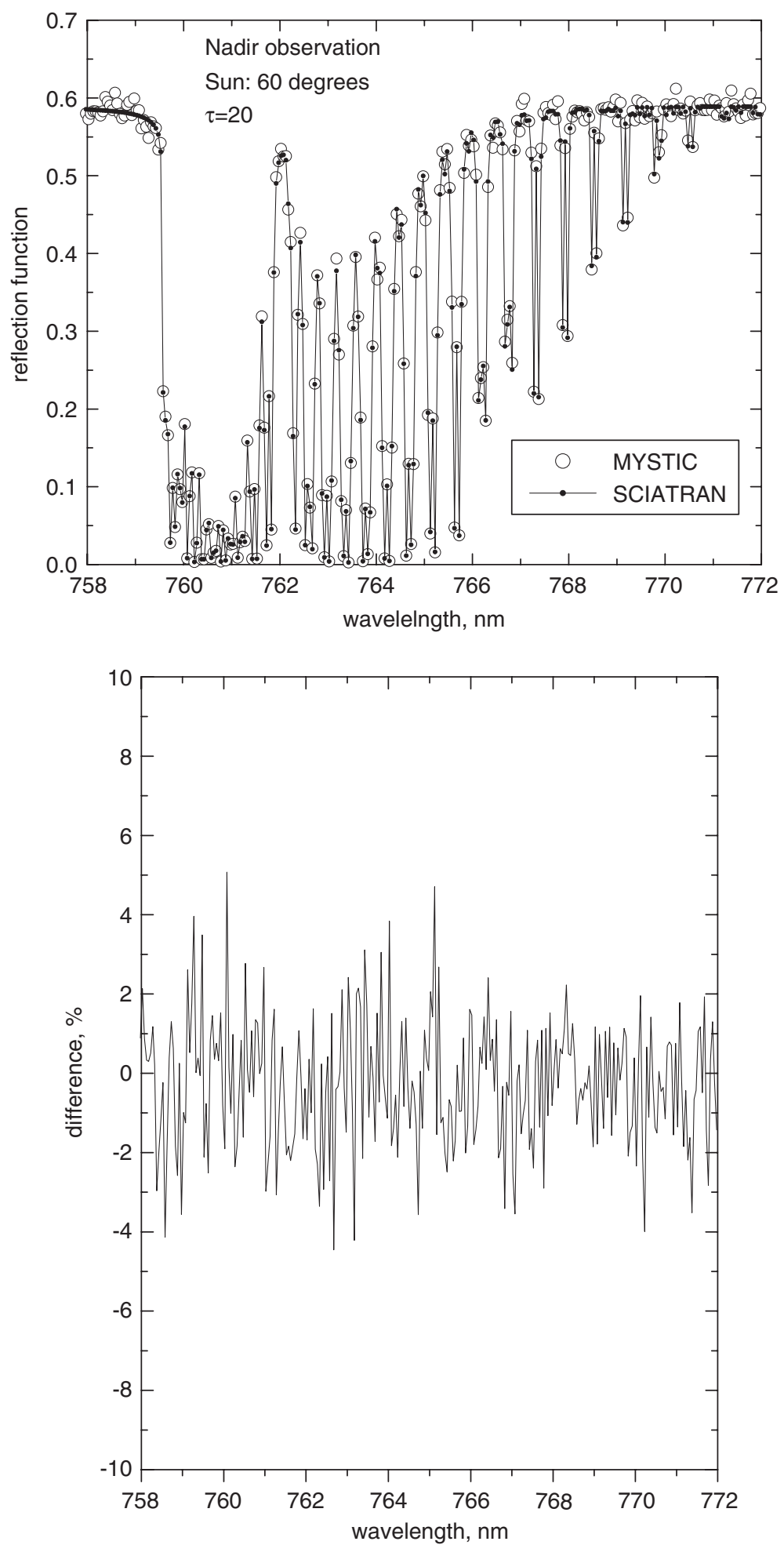

Fig. 1. (Top) Reflection function in the oxygen A-band calculated by MYSTIC (open circles) and SCIATRAN (lines with points) for solar zenith angle $60^{\circ}$, nadir observation, cloud optical thickness 20, cloud geometrical thickness $0.5 \mathrm{~km}$, and cloud top height $9.0 \mathrm{~km}$. (Bottom) Relative difference between MYSTIC and SCIATRAN. 
where $R^{\prime}=\mathrm{d} R / \mathrm{d} h . R$ is measured at several wavelengths $\lambda_{i}(i=1,2, \ldots, n)$ in the oxygen A-band. We therefore replace the scalar quantity $R$ by the vector $\vec{R}_{\text {mes }}$ with components $\left(R\left(\lambda_{1}\right), R\left(\lambda_{2}\right), \ldots, R\left(\lambda_{n}\right)\right)$. Eq. (2) can then be written in the following vector form:

$$
\vec{y}=\vec{a} x
$$

where $\vec{y}=\vec{R}_{\text {mes }}-\vec{R}\left(h_{0}\right), \vec{a}=\vec{R}^{\prime}\left(h_{0}\right)$, and $x=h-h_{0}$. Note that both measurement and model errors are contained in Eq. (3).

The solution $\hat{x}$ of the inverse problem is obtained by minimizing the cost function $\Phi=\|\vec{y}-\vec{a} x\|^{2}$ where \|\| is the norm in the Euclid space of the correspondent dimension. The value of $\hat{x}$ where the function $\Phi$ assumes its minimum can be written as

$$
\hat{x}=\frac{(\vec{y}, \vec{a})}{(\vec{a}, \vec{a})},
$$

where (,) denotes a scalar product in Euclid space. With knowledge of the measured spectral reflection function $R_{\text {mes }}$ and the calculated reflection function $R\left(h_{0}\right)$ and its derivative $R^{\prime}\left(h_{0}\right)$ at several wavelengths, the value of the CTH can be found from Eq. (4) and the equality: $h=\hat{x}+h_{0}$. The value of $h_{0}$ is set to $1.0 \mathrm{~km}$, which is a typical value for low-level clouds. To minimize the influence of calibration errors and of a priori unknown atmospheric conditions, reflectances and their derivatives were normalized to their respective values at $\lambda^{*}=758 \mathrm{~nm}$ (just outside the oxygen A-band), e.g., $R(h, \lambda) / R\left(h, \lambda^{*}\right), R\left(h_{0}, \lambda\right) / R\left(h_{0}, \lambda^{*}\right)$, and $(R(h, \lambda) /$ $\left.R\left(h, \lambda^{*}\right)\right)^{\prime}$. The retrieved data are thus only sensitive to relative differences, rather than to absolute values.

The reflection function $R$ in the oxygen A-band depends not only on the CTH $h$ but also on the cloud geometrical thickness $l$ [32]. Due to multiple scattering, the pathlengths of photons are enhanced in the cloud and any absorber inside the cloud is more effective than outside [24]. Therefore, the value of $\hat{x}$ depends on the assumed cloud geometrical thickness $l$. Accordingly, the cost function depends on the choice of $l$ also. To retrieve both cloud geometrical thickness $l$ and cloud height $h$ simultaneously we use the same technique as described above but generalized to the case of two unknown parameters [32].

In the next step we need a model for the spectral reflectivity $R$. SACURA is based on the approximate representation of $R$ by the following equation [16]:

$$
R=R_{0}+T_{1} R_{\mathrm{c}} T_{2},
$$

where $R_{0}$ gives the reflection function of the atmosphere above the cloud, $R_{\mathrm{c}}$ is the cloud reflection, and $T_{i}(i=1,2)$ are the transmission from the sun to cloud top and from cloud top to the satellite, respectively. Approximate equations for all functions in Eq. (5) are given elsewhere [16]. The comparison of the TOA reflectance calculated by SCIATRAN and approximated by Eq. (5) is shown in Fig. 2 (top) for a geometrical thickness $l=1 \mathrm{~km}$ and various cloud top altitudes. As one can see, the oxygen A-band is more shallow for higher clouds. This can be easily understood taking into account screening of tropospheric oxygen by high clouds. This general feature is exploited by the CTH retrievals in this work. For the instrument spectral response function we assumed a Gaussian function with full width at half maximum (FWHM) $0.45 \mathrm{~nm}$, which is close to that of SCIAMACHY. This response function is used in all calculations presented below. We found that the error of Eq. (5) is smaller than 5\% (see Fig. 2(top)) for cloud optical thickness larger than 5. Therefore, Eq. (5) can be used as a basis for the retrieval procedure for the case of optically thick clouds. Thin clouds are out of the scope of this paper.

The performance of SACURA for the retrieval of the $\mathrm{CTH} h$ and the cloud geometrical thickness $l$ is shown in Fig. 3 where we present the uncertainty of the retrieved CTH $h$ (a) and the cloud geometrical thickness $l$ (b) as function of $h$. For this sensitivity study, the TOA reflectance has been calculated with SCIATRAN at $l=0.5 \mathrm{~km}, l=1 \mathrm{~km}$, and $l=2 \mathrm{~km}$ assuming $100 \%$ cloud cover and various CTHs as shown in Fig. 2. All other conditions were the same as for the calculations shown in Fig. 2.

We found that the CTH error is smaller than $1 \mathrm{~km}$. The uncertainty of the geometrical thickness $l$ is also smaller than $1 \mathrm{~km}$, except for thin clouds with a geometrical thickness less than $0.5 \mathrm{~km}$. Such an accuracy is comparable to the accuracy of infrared sounders (e. g., $1.5 \mathrm{~km}$ as specified by [8]). The uncertainty decreases considerably if one of the parameters ( $h$ or $l$ ) is known in advance. In particular, one can use the correlation between $l$ and cloud optical thickness $\tau$ to estimate $l$. Such a relationship is for example given by Feigelson [7]. 

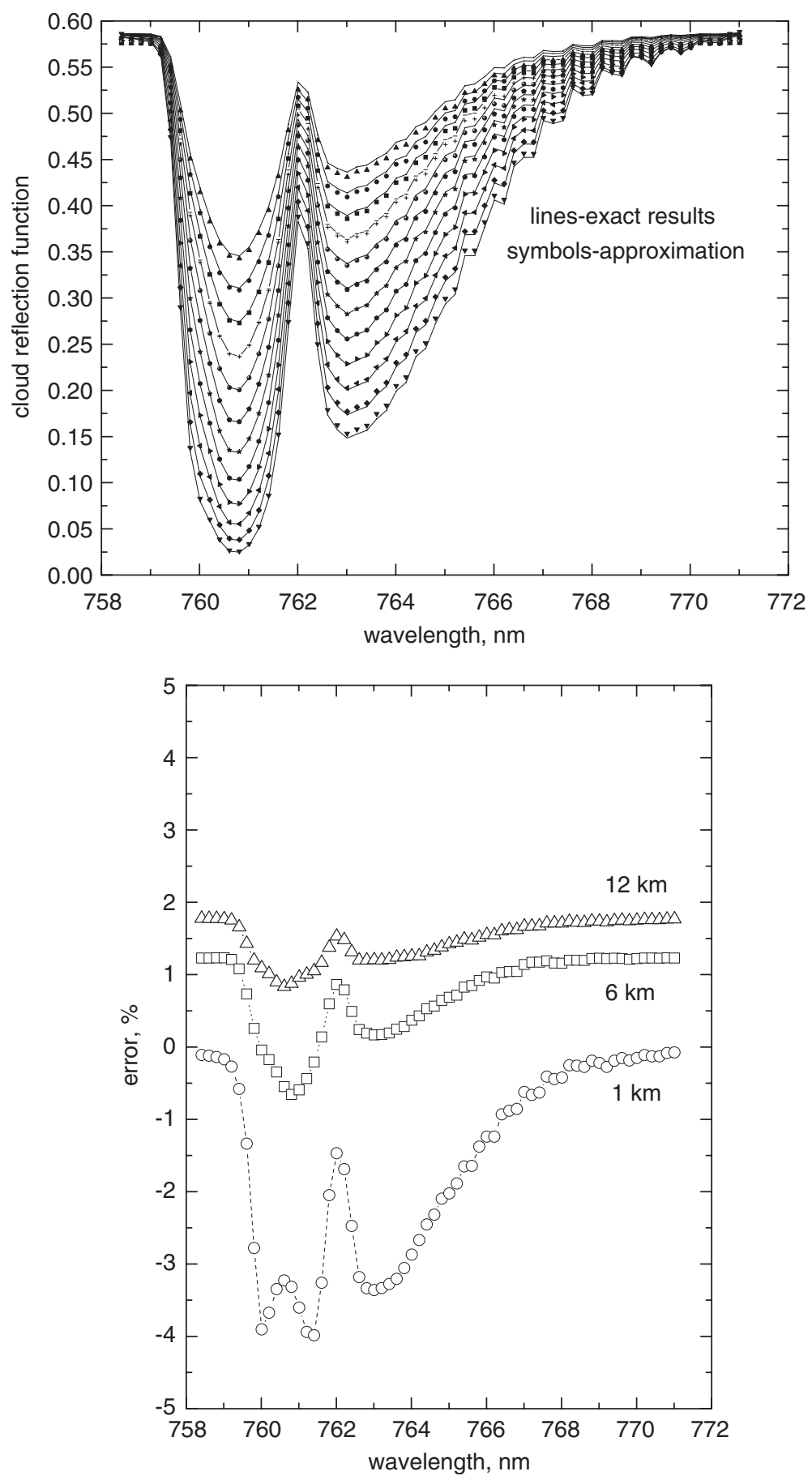

Fig. 2. (Top) Reflection function calculated by SCIATRAN (lines) and the modified exponential approximation given by Eq. (5) (symbols) for a cloud with optical thickness 20 and geometrical thickness $1 \mathrm{~km}$ for different cloud top heights from $1 \mathrm{~km}$ (lowermost curve) to $12 \mathrm{~km}$ (uppermost curve) in steps of $1 \mathrm{~km}$. The solar zenith angle is $60^{\circ}$ and the observation is at nadir. (Bottom) Errors of the modified exponential approximation compared to the SCIATRAN simulation for cloud top heights 1,6 , and $12 \mathrm{~km}$. 

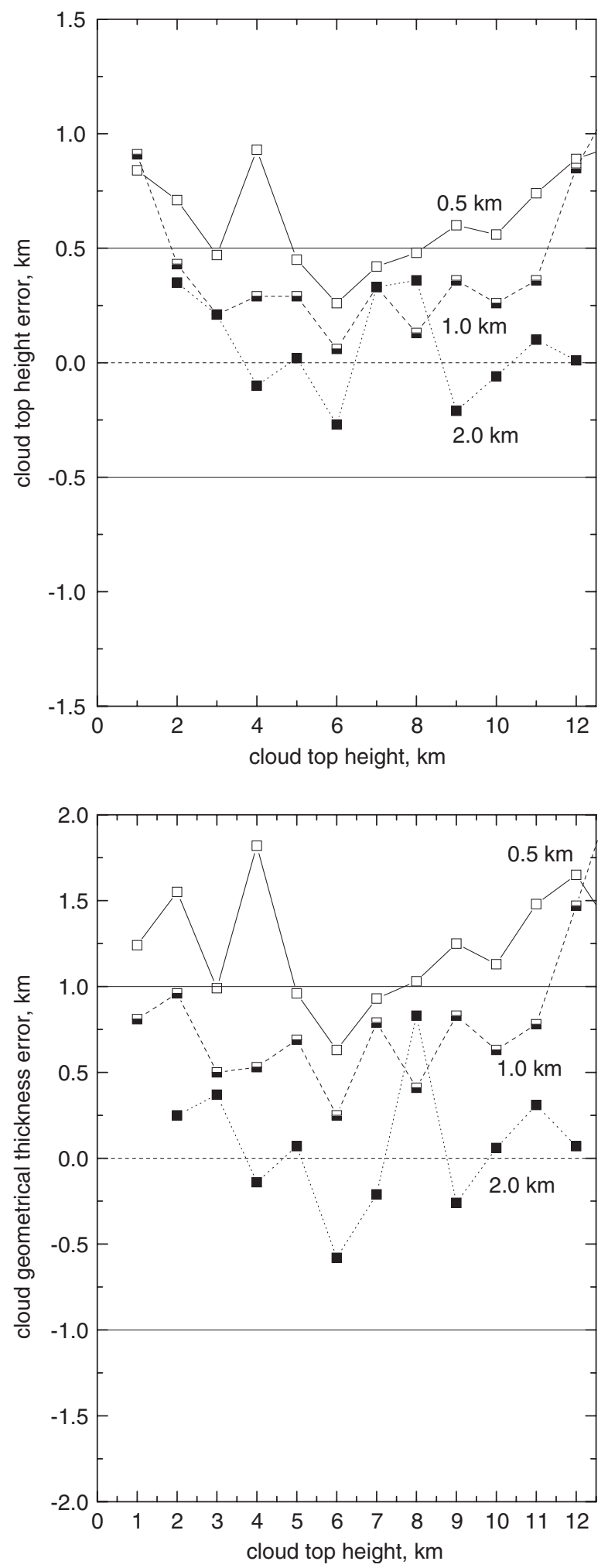

Fig. 3. Errors of the retrieved cloud top height (a) and cloud geometrical thickness (b) as functions of the cloud top height retrieved by SACURA for cloud geometrical thicknesses $0.5,1.0$, and $2.0 \mathrm{~km}$. The solar zenith angle is $60^{\circ}$ and the observation is at nadir. The cloud optical thickness is 20 . 
We obtain from their Fig. 4.2 that the extinction coefficient is typically $30 \mathrm{~km}^{-1}$ and get

$$
l=\frac{\tau}{30},
$$

where $l$ is in $\mathrm{km}$. The value of $\tau$ is easily determined from measurements outside the gaseous absorption band, either by a lookup-table approach or using an analytical approximation [15]. Note that Eq. (6) must be used with caution due to the great variability of cloud properties in space and time. Further details of SACURA are given by Rozanov and Kokhanovsky [32].

\section{Retrieval of cloud parameters for broken cloud conditions}

\subsection{The independent pixel approximation}

Fig. 4 shows a MYSTIC calculation of the reflectance of a simple 2D cloud. The cloud was embedded into the atmosphere described above. It extends horizontally from 5 to $10 \mathrm{~km}$ in a model domain of $15 \mathrm{~km}$ with periodic boundary conditions. The vertical extension was from 0.5 to $1 \mathrm{~km}$, assuming a constant extinction coefficient $40 \mathrm{~km}^{-1}$, resulting in a vertically integrated optical thickness of 20 . In $y$-direction (perpendicular to the paper) the properties are constant.

Fig. 4 illustrates the effects of 3D radiative transfer which are most pronounced for low sun: here the cloud sides intercept a considerably larger fraction of the radiation than a plane-parallel cloud. Part of this radiation is scattered into the upward direction which causes a strong peak at the sunlit side of the cloud. For high sun, part of the photons leaks out of the side of the clouds for which reason the reflectance is smaller than the 1D approximation. It is immediately clear that these effects will become more dominant for clouds which are "smaller" in the horizontal directions. For larger cloud elements, the reflectance approaches its 1D value towards the cloud center; if the cloud elements are smaller, the edge effects dominate. In fact, for cubic cloud elements $\left(1 \times 1 \times 1 \mathrm{~km}^{3}\right)$ we obtained differences of up to a factor of 2 between the 3D and independent pixel (area averaged 1D calculation, see below) results for the domain-averaged reflectivity (not shown here). Further discussions of these effects are given e.g. by Varnai and Marshak [37,38] and Nikolaeva et al. [29].

Satellite retrievals need two simplifying assumptions: (a) for technical reasons, net horizontal flux between individual pixels is neglected (IPA); (b) the clouds within each pixel are assumed homogeneous (plane-parallel approximation). While (a) is important for instruments with high spatial resolution (e.g. MODIS), (b) becomes more relevant for instruments with low spatial resolution like GOME and SCIAMACHY. Net horizontal flux may be neglected for these instruments and hence we will restrict the discussion on the second effect.

Therefore, we are not so much interested in the spatial backscattered light distribution as shown in Fig. 4 but rather in the domain-averaged reflectance. Even for the near-plane-parallel clouds used as input for Fig. 4 we find that the domain-averaged reflectance deviates from the homogeneous value: in particular for high sun the reflectance is smaller than that of a homogeneous cloud due to loss of photons leaking out of the cloud sides and never reaching the detector.

The spectral SCIAMACHY or GOME observations give an indication about cloud fraction within each pixel; however, they don't provide any detailed information about the horizontal distribution of the clouds. A more detailed information may be obtained from high-resolution instruments on the same satellite platform, e.g. ATSR-2 on ERS-2 or AATSR on ENVISAT. Computational time constraints of operational retrievals, however, currently prohibit the use of more detailed information than cloud cover. Therefore we have to address the question how sub-pixel cloud inhomogeneity affects the TOA nadir reflectances and in particular the retrieval results. Assuming that we know the cloud fraction of a pixel, we test the accuracy of the IPA to calculate domain-average reflectances:

$$
R=c R_{\mathrm{c}}+(1-c) R_{\mathrm{a}}
$$

irrespectively to the horizontal distribution of the clouds. Here $c$ is the cloud fraction which is the ratio of the area covered by clouds to the whole area of the ground scene studied, $R_{\mathrm{a}}$ is the clear sky reflection function and $R_{\mathrm{c}}$ is the cloud reflection function. To check the applicability of Eq. (7) we calculated $R, R_{\mathrm{a}}$, and also $R_{\mathrm{c}}$ 

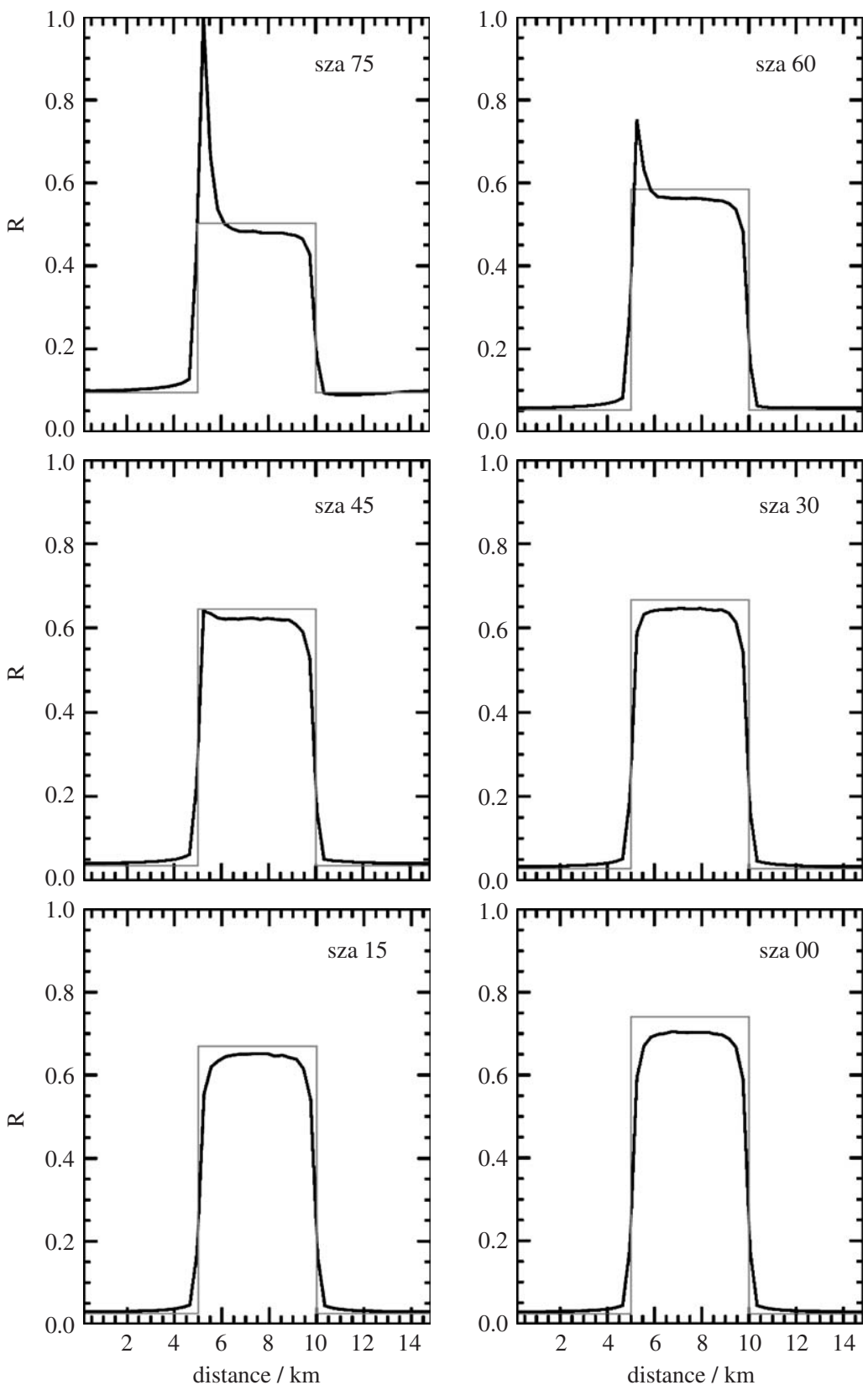

Fig. 4. 3D radiative transfer calculation of the reflectance for simple $2 \mathrm{D}$ cloud, extending from $5-10 \mathrm{~km}$ in the horizontal and from 0.5 to $1.0 \mathrm{~km}$ in the vertical, for different solar zenith angles (sza). The position of the sun is to the left. The straight lines are the respective onedimensional calculations which are basis of the independent pixel approximation.

with MYSTIC for different cloud fractions $c$ for a SCIAMACHY ground pixel of $60 \times 30 \mathrm{~km}^{2}$. As cloud model we adopted a random distribution of cuboids with dimensions $1 \times 1 \times 0.5 \mathrm{~km}^{3}$ as shown in Fig. 5. The model resolution was $0.25 \mathrm{~km}$, resulting in a grid size of $240 \times 120$ pixels. Each cloud cuboid is randomly placed in the domain and cuboids were allowed to overlap. The vertically integrated optical thickness was 20 (also for the overlapping regions). One should be aware that this is kind of a worst-case scenario: cuboid clouds have long been known to produce strong 3D effects, e.g. McKee and Cox [26]. Nevertheless, this seemingly simple cloud model is actually a good representation of a shallow cumulus cloud field. For our analysis we placed the clouds between 0.5 and $1 \mathrm{~km}$, and between 8.5 and $9 \mathrm{~km}$. In particular for the latter 

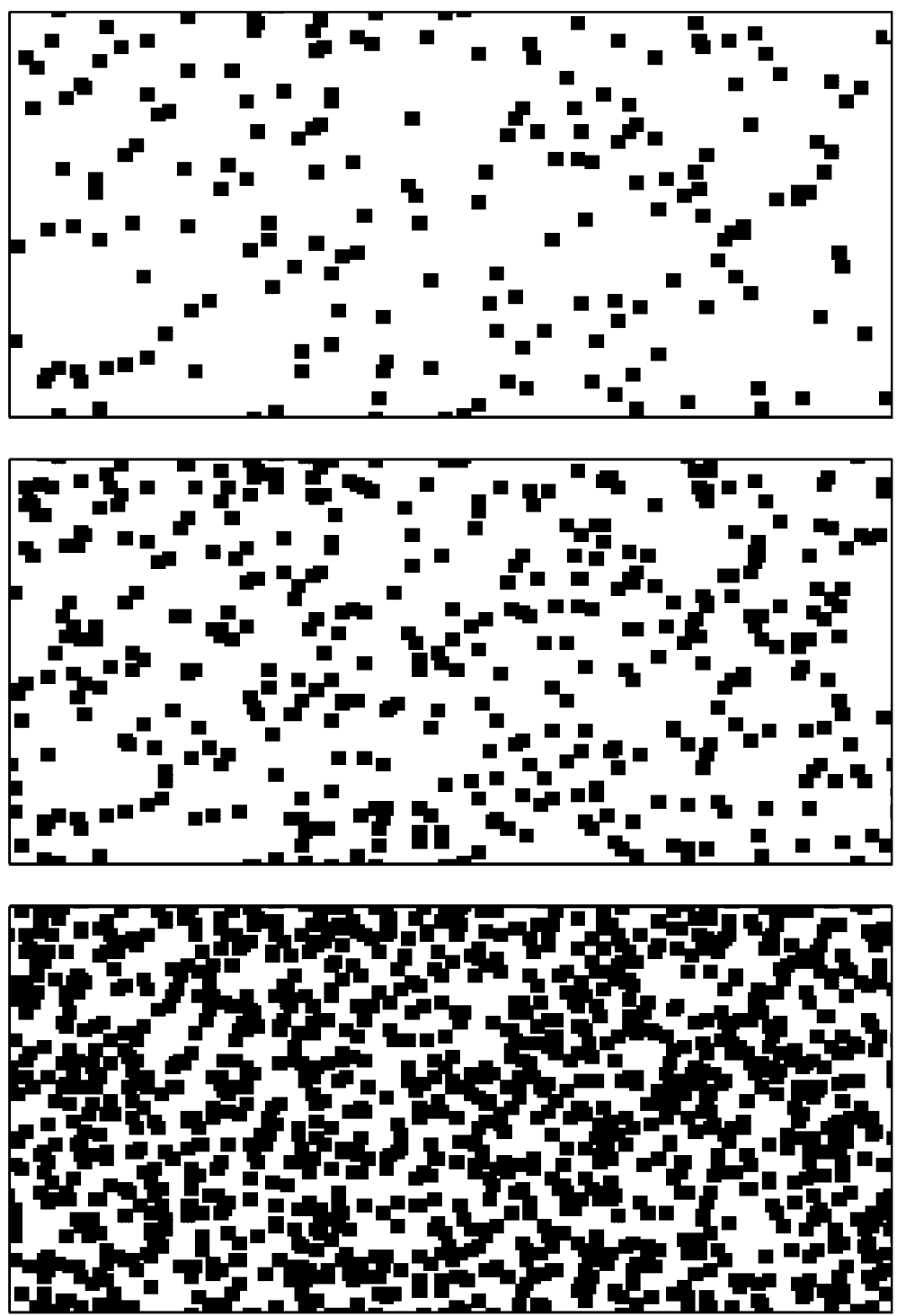

Fig. 5. Cloud model used for this study: The $x y$-cross section of the model domain shows a set of randomly placed cuboids, for cloud fractions 0.1 (top), 0.2 (middle), and 0.5 (bottom). The field contains $240 \times 120$ pixels of $0.25 \times 0.25 \mathrm{~km}^{2}$, resulting in a domain size of $60 \times 30 \mathrm{~km}^{2}$ (the SCIAMACHY IFOV). Each cuboid includes $4 \times 4$ pixels.

arrangement, this is a highly idealized setup. Namely, clouds at these altitudes are usually either cirrus clouds (which are excluded from the retrieval presented here) or high-reaching convective clouds. In order to demonstrate the abilities and limitations of the CTH retrieval, however, we decided to use the simplified but challenging cloud model described above. Fig. 6 shows the MYSTIC calculation of the reflection function for different cloud fractions between 0.1 and 1 for a solar zenith angle $\vartheta_{0}=60^{\circ}$.

For the retrieval of CTH we need the reflectance of the cloudy part of the scene. Assuming that the cloud fraction $c$ and the cloudless sky reflectance $R_{\mathrm{a}}$ are known, we obtain from the IPA, Eq. (7):

$$
R_{\mathrm{c}}=c^{-1}\left(R_{\mathrm{mes}}-R_{\mathrm{a}}\right)+R_{\mathrm{a}},
$$

where $R_{\text {mes }}$ is the observed radiance. The error $\varepsilon$ introduced by the IPA for $R_{\mathrm{c}}$ as derived from Eq. (8) is shown in Fig. 7. This error is defined as $\varepsilon=100\left(1-R_{\mathrm{c}}^{\mathrm{IPA}} / R_{\mathrm{c}}^{\mathrm{MC}}\right)$, where $R_{\mathrm{c}}^{\mathrm{MC}}$ is the "exact" cloudy sky reflectance, and $R_{\mathrm{c}}^{\mathrm{IPA}}$ is the cloudy sky reflectance approximated by Eq. (8) ( $R_{\mathrm{a}}$ is the "exact" cloudless reflectance and $R_{\text {mes }}$ is 


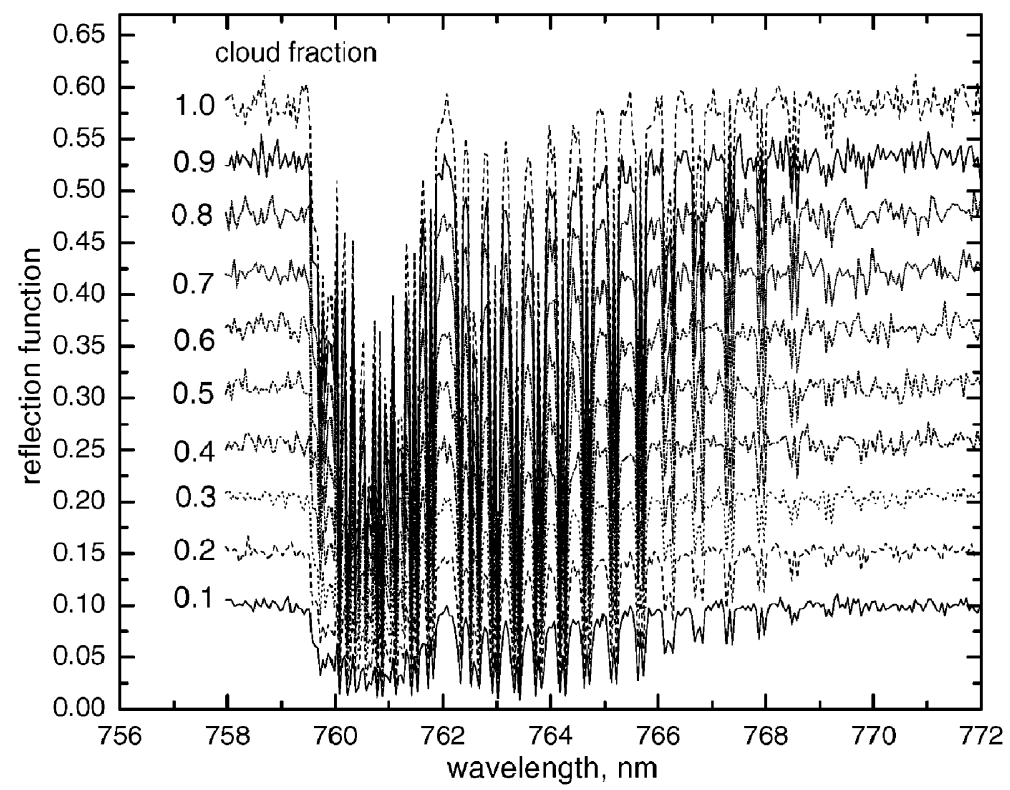

Fig. 6. Reflection function in the oxygen A-band calculated by MYSTIC for different cloud fractions.

the "exact" 3D reflectance). Fig. 7 shows this error as a function of wavelength for solar zenith angles of 0,30 , 45 , and $60^{\circ}$. We find that the uncertainty of the IPA for $R_{\mathrm{c}}$ is strongly dependent on solar zenith angle. Interestingly, for this cloud geometry the uncertainty is largest for high sun which can be explained by the results presented in Fig. 4. Namely, for high sun and nadir observation geometry the reflectivity is reduced due to photons leaking out of the cloud sides. The error is largest for small cloud fractions and vanishes in the plane-parallel limits at $c=0$ (cloudless) and $c=1$ (overcast homogeneous cloud). The best agreement is found at solar zenith angle $60^{\circ}$ while the differences increase again for larger angles (not shown here). The explanation for the small value at $60^{\circ}$ is a cancellation of errors: the reflectance is decreased due to loss of photons through the cloud sides, as for high sun; this decrease is compensated by a gain due to photons intercepted by the illuminated cloud sides and scattered in the upward direction as shown in Fig. 4 at $\vartheta_{0}=75^{\circ}$. We found that errors are positive in most cases $\left(R_{\mathrm{c}}^{\mathrm{IPA}}<R_{\mathrm{c}}^{\mathrm{MC}}\right)$.

A very important feature to note is that in all cases the error shows only little spectral dependence. For retrievals that use the absolute value of the reflectance, like, e.g., for optical thickness retrievals, we expect large errors due to the systematic bias of the reflectance. For low sun, where the 3D reflectance is systematically higher than the $1 \mathrm{D}$ approximation (see, e.g., Fig. 4 at $\vartheta_{0}=75^{\circ}$ ) observations show that the actual reflectance may take values well outside the range of reflectivities "allowed" by the IPA [17]. SACURA, however, is mainly based on spectral ratios. Hence we expect the influence of the reflectance bias to be considerably smaller. In the next section we give a detailed analysis of the uncertainty of the SACURAretrieved CTHs, by applying the retrieval to the synthetic reflectances presented in this section.

\subsection{Retrievals using the synthetic top-of-atmosphere nadir reflectance}

By applying SACURA to "exact" synthetic spectral reflectances we are able to study the uncertainty of the retrieved cloud products. Other than in real observations, where only the reflectances are known, here we know reflectances plus the real cloud properties. If the inverse problem is solved in a correct way, the SACURA output should coincide with the MYSTIC input. As SACURA is based on approximate radiative transfer theory [16] and on the IPA (see Eq. (8)), we expect some deviations.

Fig. 8 shows the error of the SACURA-derived CTH for a high optically thick cloud $(\tau=20)$ positioned between 8.5 and $9.0 \mathrm{~km}$ as a function of cloud fraction and for solar zenith angles 0,30 , and $60^{\circ}$. The errors decrease with increasing solar zenith angle which may be explained by the smaller uncertainty of the IPA at 

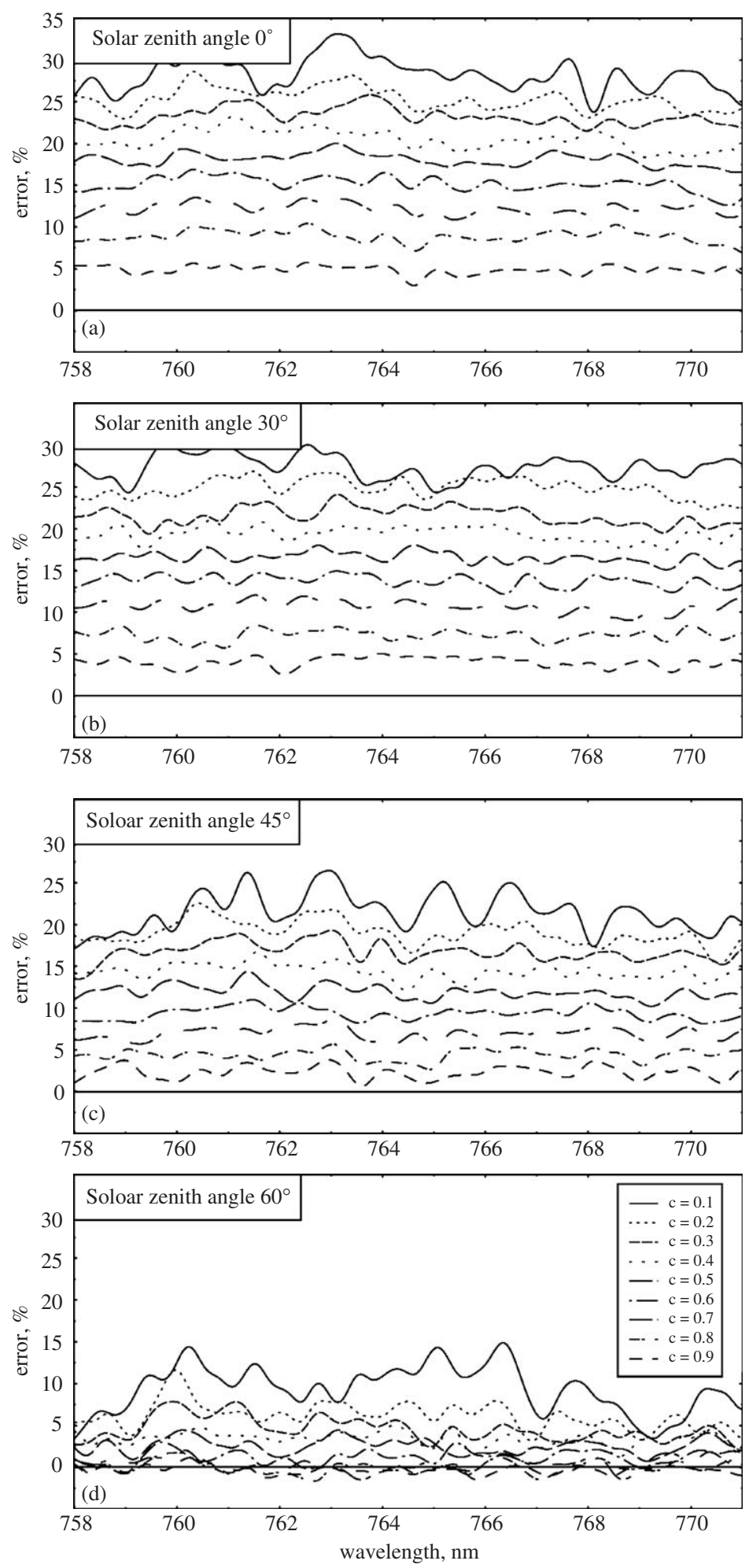

Fig. 7. Uncertainty of the independent pixel approximation for the calculation of the cloudy sky reflectance $R_{\mathrm{c}}$ using Eq. (8) as compared to the exact result for solar zenith angles $0^{\circ}$ (a), $30^{\circ}$ (b), $45^{\circ}$ (c), and $60^{\circ}$ (d). 


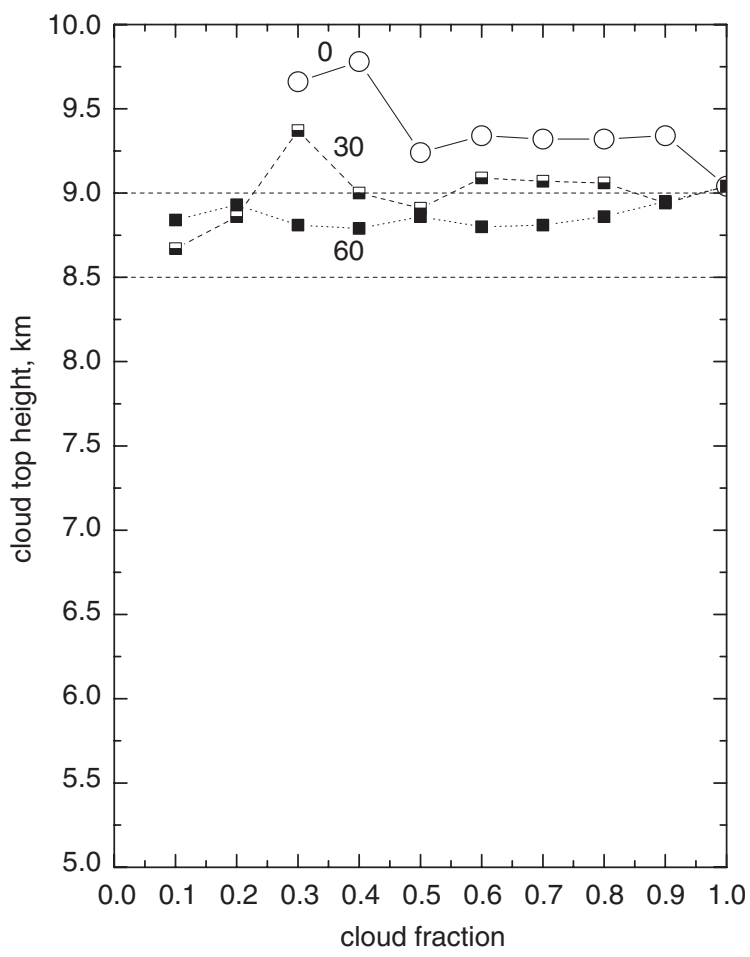

Fig. 8. Cloud top height retrieved by SACURA for a "true" cloud top height $9 \mathrm{~km}$ and geometrical thickness $0.5 \mathrm{~km}$ as function of the cloud fraction for solar zenith angles $0^{\circ}, 30^{\circ}$, and $60^{\circ}$. The "true" cloud optical thickness is 20 . In the retrieval procedure a cloud geometrical optical thickness of $0.67 \mathrm{~km}$ was assumed.

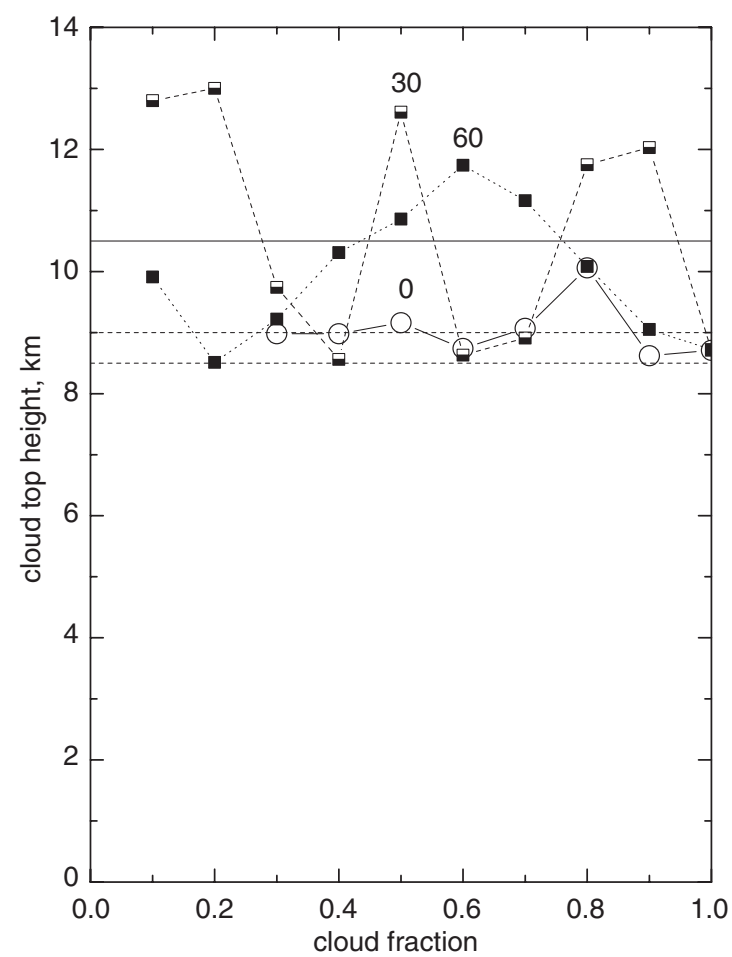

Fig. 9. Cloud top height retrieved by SACURA for a "true" cloud top height $9 \mathrm{~km}$ and geometrical thickness $0.5 \mathrm{~km}$ as function of the cloud fraction for solar zenith angles $0^{\circ}, 30^{\circ}, 45^{\circ}$, and $60^{\circ}$. In contrast to Fig. 8, the cloud geometrical thickness was not prescribed using Eq. (6) but retrieved. 

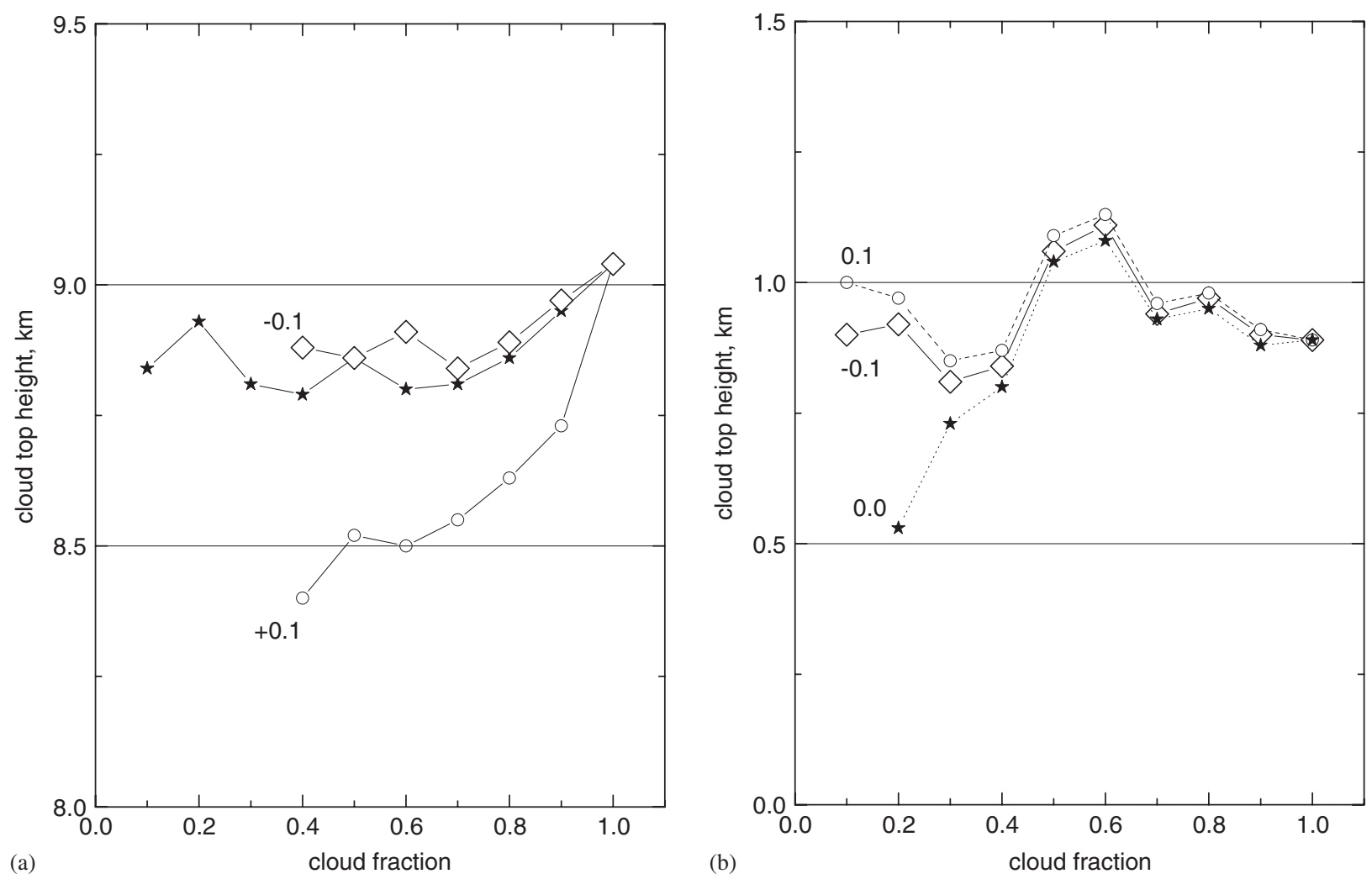

Fig. 10. Cloud top height retrieved by SACURA (stars) for "true" cloud top height $9 \mathrm{~km}$ (a) and $1 \mathrm{~km}$ (b) for a geometrical thickness $l=0.5 \mathrm{~km}$ as function of the cloud fraction. Circles and squares give the values of the cloud top height, assuming a biased cloud fraction $\bar{c}=c \pm 0.1$. The solar zenith angle is $60^{\circ}$ and the observation is at nadir. The "true" cloud optical thickness is 20 . In the retrieval procedure a cloud geometrical optical thickness of $0.67 \mathrm{~km}$ was assumed.

$60^{\circ}$ as shown in Fig. 7. The errors are generally smaller than $0.75 \mathrm{~km}$ for all solar zenith angles studied. Note that a constant value $l=0.67 \mathrm{~km}$ was assumed for the cloud geometrical thickness (see Eq. (6)) in the retrieval shown in Fig. 8. If the value of $l$ is not determined independently (e.g., using Eq. (6)) but rather it is calculated in the retrieval procedure simultaneously with $h$, the errors increase as shown in Fig. 9. The different behavior of the accuracy with respect to the solar zenith angle shown in Fig. 9 (as compared to Fig. 8) is due to the compensation of the errors of the approximate equations used in SACURA [16] and the IPA (see Fig. 7). The conclusion from our calculations is that it is advisable to run SACURA for a fixed cloud geometrical thickness, e.g., as estimated from Eq. (6). Our results indicate that even if $l$ is not precisely known, the uncertainty of the retrieved values of $h$ is considerably reduced if the cloud geometrical thickness is available from independent observations.

The cloud fraction $c$ can be obtained using additional measurements with higher spatial resolution (e.g., the broad band measurements of the polarization measurements devices [36] of SCIAMACHY and GOME, or using ATSR-2 and AATSR placed on the same satellite platforms). In the following we show the influence of the uncertainties in the cloud fraction $c$ on the CTH retrievals. Fig. 10 shows the impact of an error of 0.1 in the cloud fraction. The retrievals have again been performed for a fixed geometrical thickness $l=0.67 \mathrm{~km}$. The cloud reflectance is calculated from Eq. (8). For the sensitivity study, we used the actual ("true") cloud fraction as well as a biased one by \pm 0.1 . The figure illustrates that the uncertainty in the cloud fraction $\Delta c= \pm 0.1$ does not lead to a considerable increase in the retrieval error for high and low clouds for the cases studied. Absolute errors of the order 0.1 are common in retrievals of $c$ using, e.g., polarization measurement devices incorporated in GOME and SCIAMACHY [36].

The uncertainty of the retrieved CTH is generally larger if the value of $l$ is determined simultaneously in the retrieval procedure (see Fig. 11). However, this is not related to the uncertainty in the cloud fraction but rather 


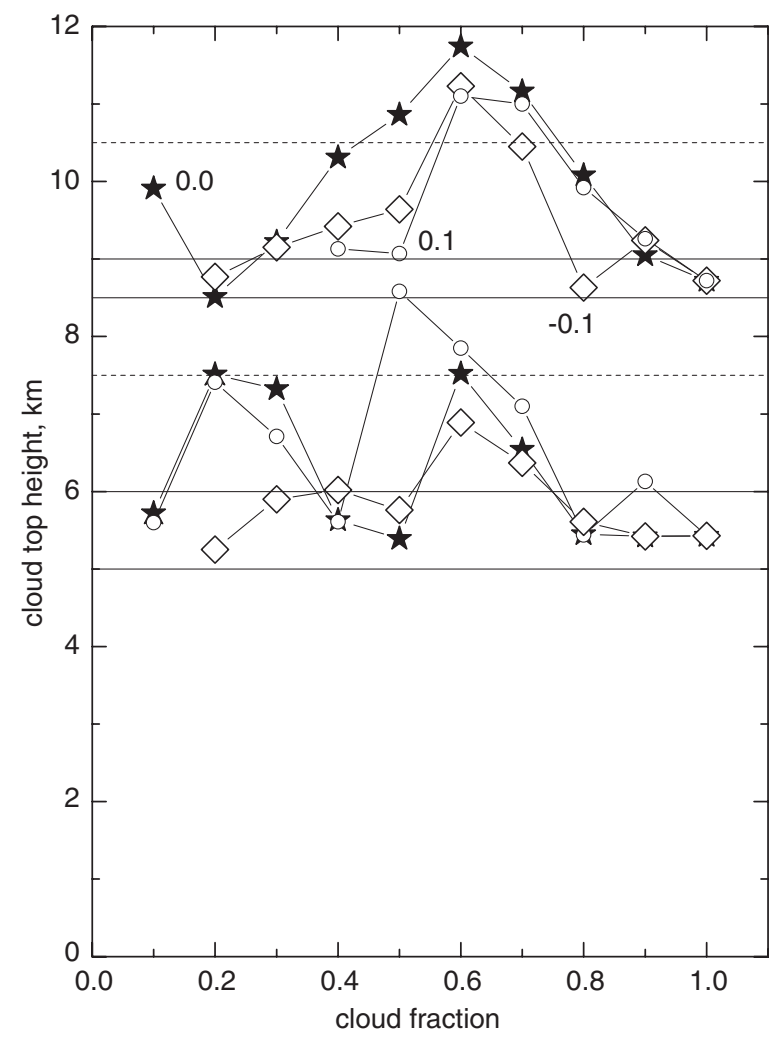

Fig. 11. Cloud top height retrieved by SACURA (stars) for a "true" cloud top height $9 \mathrm{~km}$ and cloud bottom height $8.5 \mathrm{~km}$ as function of cloud fraction. Circles and squares give the values of the cloud top height (CTH) for the biased cloud fraction $\widehat{c}=c \pm 0.1$. The solar zenith angle is $60^{\circ}$ and the observation is at nadir. The "true" cloud optical thickness is 20 . Results for the case $h=6 \mathrm{~km}, l=1 \mathrm{~km}$ are given as well (lower set of curves). In contrast to Fig. 10, the cloud geometrical thickness was not prescribed but retrieved.

due to problems associated with simultaneous determination of the pair $(h, l)$ from observations in the oxygen A-band alone [16]. Interestingly, inaccurate values of the cloud fraction $c$ may lead to a compensation of errors of SACURA (see Fig. 11). This is related to the fact that the error in $c$ affects mostly the absolute radiance but not the spectral feature used in the $\mathrm{CTH}$ retrievals. The cloud optical thickness can be derived from measurements performed outside the $\mathrm{O}_{2}$ A-band. We expect much larger errors in the retrieved values of $\tau$ compared to $h$ for broken cloud fields. Fig. 12 shows that this is actually the case. These differences are best explained by the fact that the retrieval of $\tau$ depends on absolute values of the reflection function while for the CTH determination we use ratios of reflectances and not absolute values of $R$.

\section{Conclusions}

Modern satellite trace gas retrieval algorithms must be improved with respect to the incorporation of detailed information on clouds present during the measurements. The restriction to completely cloudless pixels reduces the information content of measurements considerably. The main cloud parameters needed, e.g., for the determination of the total ozone, are the cloud fraction, the $\mathrm{CTH}$, and the cloud optical thickness. These parameters can be determined using cloud retrieval algorithms. An important requirement for such algorithms for the application to studies of atmospheric chemical composition are their accuracy and speed. SACURA is based on asymptotic analytical equations, and, therefore, it satisfies the speed constrains. Its accuracy is also satisfactory for problems related to the trace gas vertical column determination. We summarize that SACURA is capable to retrieve cloud top altitudes not only for overcast scenes (see Fig. 3) as it was stated before [32] but also for partially cloudy scenes, if the cloud fraction $c$ is obtained from independent sources, e.g., from the polarization measurement devices of GOME and SCIAMACHY [36]. 


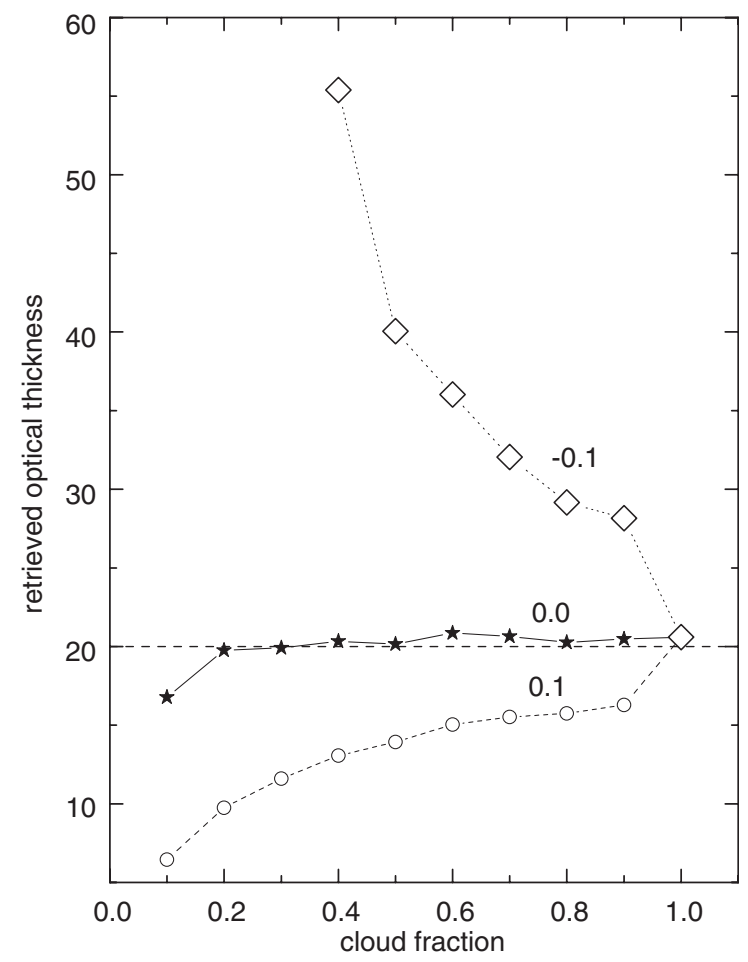

Fig. 12. Cloud optical thickness retrieved by SACURA (stars) for a "true" cloud optical thickness 20. Circles and squares give the values of the cloud top height assuming a modified cloud fraction $\widehat{c}=c \pm 0.1$ in the retrieval. The solar zenith angle is $60^{\circ}$ and the observation is performed at nadir geometry.

In addition to the standard version of SACURA [32], the cloud reflectance is determined using the IPA based on the cloud fraction and climatological values of the clear sky reflectance $R_{\mathrm{a} .}$. $R_{\mathrm{a}}$ may also be obtained from minimum values of $R_{\mathrm{a}}$ for a given pixel or area, see e.g., by Koelemeijer et al. [14]. This is of special importance for land surfaces, where the unknown surface albedo (assumed to be zero in this study) may further bias results. Accurate values of $R_{\mathrm{a}}$ are not needed for values of $c$ close to one. In this case the reflectance is dominated by the cloudy portion of the scene.

Our studies showed that 3D radiative transfer may have a considerable effect on the absolute values of the reflectance. However, the deviations between exact 3D theory and the IPA were relatively constant over the spectral range of the oxygen A-band. Hence, the retrieved optical thickness (based on absolute reflectance values) is strongly affected by $3 \mathrm{D}$ radiative effects while the impact on the determination of CTH (based only on spectral ratios) is only slightly sensitive to $3 \mathrm{D}$ effects.

The error of the cloud top altitude determination using SACURA for the overcast clouds was smaller than $1 \mathrm{~km}$, if both cloud geometrical thickness and CTH are determined in the retrieval procedure. The error depends on the cloud geometrical thickness and is larger for thinner clouds (see Fig. 3). We also found that the error is highly sensitive to a priori assumptions on the cloud geometrical thickness and also on the solar zenith angle and the cloud fraction (compare Figs. 8 and 9). It is considerably reduced, if the value of the cloud geometrical thickness is obtained from independent measurements. This indicates a possibility to retrieve the cloud geometrical thickness and also cloud bottom height from reflectance measurements in the oxygen A-band, e.g., if the CTH is determined by a lidar [19]. In most broken cloud cases the error of SACURA was smaller than $1.5 \mathrm{~km}$ which is close to typical errors of IR measurements as applied to the determination of cloud altitudes from space. For instance, Frey et al. [8] reported that the $\mathrm{CO}_{2}$ slicing method retrieved cloud heights to within 0.5 and $1.5 \mathrm{~km}$ of the lidar heights in $32 \%$ and $64 \%$ of the cases, respectively. Naud et al. [28] demonstrated that MODIS derived CTH altitudes differ from that of a ground-based radar by $1-2 \mathrm{~km}$. Minnis 
et al. [27] have shown that IR measurements are capable to determine altitudes of clouds with the error less than $0.5 \mathrm{~km}$ for thick extended clouds and to within $1.0 \mathrm{~km}$ for thin clouds.

We believe that the results obtained here and also the Monte-Carlo technique used are of interest for several scientific groups conceiving cloud altitude determination algorithms using backscattered radiation in gaseous absorption bands and generally for those interested in the development of accurate procedures to determine trace gas concentrations from orbiting and geostationary satellites.

\section{Acknowledgements}

This work has been supported by DFG Projects BU 688/8-1 and MA 2548/1-1.

\section{References}

[1] Bovensmann H, et al. SCIAMACHY: Mission objectives and measurement methods. J Atmos Sci 1999;56:127-50.

[2] Buchwitz M, Rozanov VV, Burrows JP. A correlated-k distribution scheme for overlapping gases suitable for retrieval of atmospheric constituents from moderate resolution radiance measurements in the visible/near-infrared spectral region. J Geophys Res 2000;D105: 15247-62.

[3] Burrows JP, et al. The global ozone monitoring experiment (GOME): mission concept and first scientific results. J Atmos Sci 1999;56: 151-75.

[4] Cahalan RF, et al. Independent pixel and Monte-Carlo estimates of stratocumulus albedo. J Atmos Sci 1994;51:3776-90.

[5] Cahalan RF, et al. The International Intercomparison of 3D Radiation Codes (I3RC): Bringing together the most advanced radiative transfer tools for cloudy atmospheres, Bull Am Meteorol Soc, 2005;86:1275-93.

[6] Davis JM, et al. Application of the Monte-Carlo method to problems in visibility using a local estimate: an investigation. Appl Opt 1985;24:3193-205.

[7] Feigelson EM, editor. Radiation in cloudy atmosphere. Leningrad: Gidrometeoizdat; 1981.

[8] Frey RA, Baum BA, Menzel WP, Ackerman SA, Moeller CC, Spinhirne JD. A comparison of cloud top heights computed from airborne lidar and MAS radiance data using CO2 slicing. J Geophys Res 1999;104(D20):24,547-55.

[9] Heidinger AK. Nadir sounding of clouds and aerosols in the A-band of oxygen, PhD thesis, Fort Collins: Colorado State University, 1998.

[10] Kneizys FX, et al. In: Abreu LW, Anderson GP, editors. The MODTRAN 2/3 report on LOWTRAN-7 model. Contract F19628-91C-0132 with Ontar Corp. MA: Phillips Lab, Hancom AFB; 1996.

[11] Koelemeijer R. Influence of clouds on ozone measurements obtained by satellite spectrometry, PhD thesis, Amsterdam Free University, Amsterdam, 2001.

[12] Koelemeijer RBA, Stammes P, Hovenier JW, De Haan JF. A fast method for retrieval of cloud parameters using oxygen A band measurements from GOME. J Geophys Res 2001;106:3475-90.

[13] Koelemeijer RBA, Stammes P, Hovenier JW, De Haan JF. Global distributions of effective cloud fraction and cloud top pressure derived from oxygen A band spectra measured by the global ozone monitoring experiment: comparison to ISCCP data. J Geophys Res 2002;107, doi:10.1029/2001JD000840.

[14] Koelemeijer RBA, de Haan JF, Stammes P. A database of spectral surface reflectivity in the range 335-772 nm derived from 5.5 years of GOME observations. J Geophys Res 2003 .

[15] Kokhanovsky, AA, Rozanov, VV, Zege, EP, Bovensmann, H, Burrows, JP. A semi-analytical cloud retrieval algorithm using backscattered radiation in 0.4-2.4 $\mu \mathrm{m}$ spectral region. J Geophys Res 2003;108, doi:10.1029/2001JD001543.

[16] Kokhanovsky AA, Rozanov VV. The physical parameterization of the top-of-atmosphere reflection function for a cloudy atmosphere - underlying surface system: the oxygen A-band case study. JQSRT 2004;85:35-55.

[17] Kokhanovsky AA, von Hoyningen-Huene W. Optical properties of a hurricane. Atmos Res 2004;69:165-83.

[18] Kokhanovsky AA, Rozanov VV, von Hoyningen-Huene W, Bovensmann H, Burrows JP, Baltink HK. The determination of cloud altitudes using SCIAMACHY onboard ENVISAT. IEEE Trans Geosci Rem Sens Lett 2004;1:211-4.

[19] Kokhanovsky AA, Rozanov VV. Determination of a cloud bottom height from a satellite. IEEE Trans Geosci Rem Sens Lett, 2005;2:280-3.

[20] Loyola D. Automatic cloud analysis from polar-orbiting satellites using neural network and data fusion techniques. IEEE Int Geosci Remote Sens Symp 2004;4:2530-4.

[21] Marchuk GI, et al. The Monte-Carlo methods in atmospheric optics. Berlin: Springer-Verlag; 1980.

[22] Mayer B. I3RC phase 1 results from the MYSTIC Monte Carlo model. I3RC Workshop Proceedings, Tucson, Arizona, November 17-19, 1999.

[23] Mayer B. I3RC phase 2 results from the MYSTIC Monte Carlo model. I3RC Workshop Proc., Tucson, Arizona, November 15-17, 2000.

[24] Mayer B, Kylling A, Madronich S, Seckmeyer G. Enhanced absorption of UV radiation due to multiple scattering in clouds: experimental evidence and theoretical explanation. J Geophys Res 1998;103:31241-54. 
[25] Mayer B, Kylling A. Technical Note: The libRadtran software package for radiative transfer calculations: Description and examples of use. Atmos Chem Phys Discuss 2005;5:1319-81.

[26] McKee TB, Cox SK. Scattering of visible radiation by finite clouds. J Atmos Sci 1974;31:1885-92.

[27] Minnis P, Smith Jr, WL, Garber DP, Ayers JK, Doelling DR. Cloud properties derived from GOES-7 for spring 1994 ARM intensive observing period using version 1.0.0 of ARM satellite data analysis program. NASA Ref. Publ. 1366, 1995.

[28] Naud C, Muller J-P, Clothiaux EE. Comparison of cloud top heights derived from MISR stereo and MODIS CO2 -slicing. Geophys Res Lett 2002;29, doi:10.1029/2002GL015460.

[29] Nikolaeva OV, Bass LP, Germogenova TA, Kokhanovsky AA, Kuznetsov VS, Mayer B. The influence of neighbouring clouds on the clear sky reflectance studied with the 3-D transport code RADUGA. JQSRT 2005;94:405-24.

[30] Rothman LS, et al. The HITRAN molecular spectroscopic database: edition of 2000 including updates through 2001. JQSRT 2003;82:5-44.

[31] Rozanov AV, Rozanov VV, Buchwitz M, Kokhanovsky AA, Burrows JP. SCIATRAN 2.0-A new radiative transfer model for geophysical applications in the 175-2400 nm spectral range. Adv Space Res 2005;36:1015-9.

[32] Rozanov, VV, Kokhanovsky, AA, Semianalytical cloud retrieval algorithm as applied to the cloud top altitude and the cloud geometrical thickness determination from top-of-atmosphere reflectance measurements in the oxygen A band. J Geophys Res 2004; 109, doi:10.1029/2003JD004104.

[33] Rozanov VV, Kokhanovsky AA, Burrows JP. The determination of cloud top altitudes using GOME reflectance spectra: multilayered cloud systems. IEEE Trans Geosci Remote Sens 2004;42:1009-17.

[34] Siewert CE. A discrete-ordinates solution for radiative transfer models that include polarization effects. JQSRT 2000;64:227-54.

[35] Stamnes K, Tsay SC, Wiscombe W, Jayaweera K. A numerically stable algorithm for discrete-ordinate-method radiative transfer in multiple scattering and emitting layered media. Appl Opt 1988;27:2502-9.

[36] Tuinder ONE, de Winter-Sorkina R, Builtjes PJH. Retrieval methods of effective cloud cover from the GOME instrument: an intercomparison. Atmos Chem Phys 2004;4:255-73.

[37] Varnai T, Marshak A. Observations of three-dimensional radiative effects that influence MODIS cloud optical thickness retrievals. J Atmos Sci 2002;59:1607-18.

[38] Varnai T, Marshak A. A method for analyzing how various parts of clouds influence each other's brightness. J Geophys Res 2003;108, doi:10.1029/2003JD003561. 